\title{
Epigeous Macrofungi of the Parque de Natureza de Noudar in Alentejo (Portugal)
}

\author{
R. LOURO ${ }^{1}$, M. CALAdO ${ }^{1}$, B. PINTO $^{2}$, C. SANTOS-SILVA ${ }^{1}$ \\ rog.louro@gmail.com \\ maria_calado@yahoo.com.br \\ bpinto@edia.pt \\ css@uevora.pt \\ ${ }^{1}$ Instituto de Ciências Agrárias e Mediterrânicas e Departamento de Biologia da \\ Universidade de Évora, Apartado 94, 7002-554 ÉVORA, Portugal \\ ${ }^{2}$ Empresa de Desenvolvimento e Infra-estruturas do Alqueva, R. Zeca Afonso, $n^{\circ} 2$, \\ 7800-522 BEJA, Portugal
}

\begin{abstract}
This inventory represents the first list of the epigeous macrofungi collected in Parque de Natureza de Noudar (Barrancos, Alentejo, Portugal). Throughout 3 years 162 taxa were registered, from which 8 are new species for Portugal and 77 for Alentejo. Some of them are considered rare species whereas others have been suggested to acquire a conservation status. Fulllength weblist on URL: http://home.dbio.uevora.pt/ css/

Keywords - fungi, holm oak, mediterranean ecosystem, Iberian Peninsula, sporocarps
\end{abstract}

\section{Introduction}

The cork-oak (Quercus suber L.) and holm-oak (Quercus rotundifolia Lam. = Quercus ilex subsp. ballota (Desf.) Samp.) stands are the most frequent agroforestry systems in Alentejo (Portugal). In this region 35\% of the total forest area is occupied by holm-oak stands. These ecosystems are known by its biodiversity, namely its richness in macrofungal species (DGF 2001). This study was conducted in the Parque de Natureza de Noudar - Barrancos $\left(38^{\circ}\right.$ $08^{\prime} \mathrm{N}$ and $6^{\circ} 59^{\prime} \mathrm{W}$ ), Alentejo - where the landscape is dominated by holmoak stands with different shrub or herbaceous composition and cover. The understory consists of mixed sclerophyllous shrubs, mainly Cistus spp., Lavandula spp., Genista spp., Myrtus communis L., Pistacia lentiscus L., and natural pasture of annual herbaceous (mostly Asteraceae, Poaceae and Fabaceae). Furthermore, this area shelters a number of rich floral communities, with a long natural history of adaptation to the Mediterranean climate and human activities.

With a total area of 994.5 ha, the Parque de Natureza de Noudar is delimited by the Ardila river and the Múrtega stream. Inserted in a classified area of the Rede Natura 2000, it forms an ecological group with other protected areas in Spain. 
The climate is Mediterranean, with rainy mild winters and hot dry summers. Mean annual rainfall is $525.6 \mathrm{~mm}$ and mean annual air temperature $15.8^{\circ} \mathrm{C}$, with a dry period from May to September (Mendes et al. 1991). The soils are predominantly Luvisols and Leptosols.

\section{Materials and Methods}

All specimens were collected from November 2004 to April 2007 in 43 plots scattered throughout the different biotopes with a total area of 1.13 ha. Collected specimens were preserved and deposited in the Évora University Herbarium (UEVH- FUNGI).

Plant nomenclature follows Franco (1971). The macrofungi's catalogue is arranged alphabetically according to order and genus. Taxonomy and nomenclature follows Kirk et al. (2001) and Kirk (2004-08). Current species distribution area was consulted in: Calonge (1998), CMUL (2002), Hernández-Crespo (2006), Pinho-Almeida \& Baptista-Ferreira (1996, 2005) and GBIF (2008). New occurrences for Alentejo are marked with $(*)$ and for Portugal $(* *)$.

\section{Results}

Over a 3 year period, 68 genera comprising 162 macrofungi species were collected. Of these, 77 represent new records to the Alentejo region, namely: Agaricus xanthodermus var. lepiotoides Maire, Agrocybe molesta (Lasch) Singer, Amanita ceciliae (Berk. \& Broome) Bas, Bovista delicata Berk. \& M.A. Curtis, Bovista dermoxantha (Vittad.) De Toni, Calvatia excipuliformis (Scop.) Perdeck, Chalciporus piperatus (Bull.) Bataille, Clavulina cinerea (Bull.) J. Schröt., Clavulina rugosa (Bull.) J. Schröt., Clitocybe font-queri R. Heim, Clitocybe obsoleta (Batsch) Quél., Coprinus alopecia Lasch, Cortinarius bulliardii (Pers.) Fr., Cystoderma amianthinum (Scop.) Fayod, Entoloma cistophilum Trimbach, Entoloma hebes (Romagn.) Trimbach, Entoloma papillatum (Bres.) Dennis, Entoloma serrulatum (Pers.) Hesler, Entoloma undatum (Fr.) M.M. Moser, Galerina vittiformis (Fr.) Earle, Geastrum elegans Vittad., Hebeloma cistophilum Maire, Helvella leucomelaena (Pers.) Nannf., Hydropus floccipes (Fr.) Singer, Hygrocybe miniata (Fr.) P. Kumm., Hygrophorus arbustivus Fr., Inocybe asterospora Quél., Inocybe calospora Quél., Inocybe cervicolor (Pers.) Quél., Inocybe flocculosa (Berk.) Sacc., Inocybe fuscidula Velen. var. fuscidula, Inocybe godeyi Gillet, Inocybe napipes J.E. Lange, Ileodictyon gracile Berk., Lactarius camphoratus (Bull.) Fr., Lactarius cistophilus Bon \& Trimbach, Leccinum corsicum (Rolland) Singer, Lepiota clypeolaria (Bull.) P. Kumm., Lepiota griseovirens Maire, Lepiota oreadiformis Velen., Lepiota pseudolilacea Huijsman, Lepiota subgracilis Wasser, Lepista sordida (Fr.) 
Singer, Lycoperdon lividum Pers., Lycoperdon nigrescens Wahlenb., Marasmius bulliardii Quél., Melanoleuca grammopodia (Bull.) Murrill, Mycena abramsii (Murrill) Murrill, Mycena aetites (Fr.) Quél., Mycena filopes (Bull.) P. Kumm., Mycena galopus var. nigra Rea, Mycena pura (Pers.) P. Kumm., Otidea alutacea (Pers.) Massee, Panaeolina foenisecii (Pers.) Maire, Panaeolus fimicola (Pers.) Quél., Parasola auricoma (Pat.) Redhead et al., Peziza arvernensis Boud., Peziza domiciliana Cooke, Peziza succosa Berk., Peziza varia (Hedw.) Fr., Phaeomarasmius erinaceus (Pers.) Scherff. ex Romagn., Pholiota highlandensis (Peck) A.H. Sm. \& Hesler, Pluteus phlebophorus (Ditmar) P. Kumm., Polyporus alveolaris (DC.) Bondartsev \& Singer, Polyporus meridionalis (A. David) H. Jahn, Psathyrella conopilus (Fr.) A. Pearson \& Dennis, Psathyrella panaeoloides (Maire) M.M. Moser, Psathyrella spadiceogrisea (Schaeff.) Maire, Rhodocybe nitellina (Fr.) Singer, Rickenella fibula (Bull.) Raithelh., Russula pseudo-olivascens Kärcher, Russula purpurata Crawshay, Tarzetta catinus (Holmsk.) Korf \& J.K. Rogers, Thelephora caryophyllea (Schaeff.) Pers., Tremella foliacea Pers., Tricholoma saponaceum (Fr.) P. Kumm. var. saponaceum and Tricholoma squarrulosum Bres.

Also, within the collected species 8 are new citations to Portugal, specifically: Clitocybe squamulosoides var. meridionalis Bon, Entoloma occultopigmentatum Noordel. \& Arnolds, Hygrophorus eburneus var. cossus (Sowerby) Quél., Leucoagaricus melanotrichus (Malençon \& Bertault) Trimbach var. melanotrichus, Mycena erubescens Höhn., Mycena flavescens Velen., Mycena rubromarginata (Fr.) P. Kumm. var. rubromarginata and Pluteus podospileus Sacc. \& Cub.

Furthermore 6 of the identified species are considered rare within the Iberian Peninsula: Agaricus porphyrizon P.D. Orton, Ileodictyon gracile, Lactarius camphoratus, Lepiota oreadiformis, Leucoagaricus melanotrichus var. melanotrichus and Phaeomarasmius erinaceus.

Additionaly Amanita caesarea (Scop.) Pers., Amanita verna (Bull.) Lam., Cortinarius orellanus Fr., Gyroporus castaneus (Bull.) Quél. and Hygrocybe conica (Scop.) P. Kumm. var. conica, also found in the Parque de Natureza de Noudar, have been referred by Prof. Dr. Francisco Calonge as threatened (SMM 2008), thus their conservation status in the Iberian Peninsula needs urgent assessment and legislation.

\section{Conclusions}

The high number of new references is undoubtedly due to the scarcity of mycological surveys in Southern Portugal, particularly in Alentejo. In fact, some of these new references correspond to widespread species in the Iberian Peninsula. On the other hand, some rare or vulnerable species occurred as 
was expected for this typical Mediterranean ecosystem known by its biodiversity.

\section{Acknowledgements}

We would like to thank EDIA (Alqueva Infrastructures Development Enterprise) for sponsoring this study. We also wish to express our gratitude to Prof. Francisco Calonge and to Prof. J. L. Baptista-Ferreira for reviewing the manuscript.

\section{Literature cited}

Calonge FD. 1998. Flora Mycologica Iberica Vol. 3. Madrid: Consejo superior de Investigaciones Científicas, CSIS. Madrid.

CMUL. 2002. Listagem preliminar de fungos agaricoides de Portugal. Centro de Micologia da Universidade de Lisboa, Lisboa.

DGF. 2001. Inventário Florestal Nacional: Portugal Continental. $3^{a}$ Revisão, Direcção-Geral das Florestas, Lisboa.

Franco JA. 1971. Nova Flora de Portugal (Continente e Açores). Lycopodiaceae - Umbelliferae, 1. Edição do autor, Lisboa.

GBIF. 2008. Global Biodiversity Information Facility Data Portal. http://www.gbif.net (last up. 06/2008).

Hernández-Crespo JC. 2006. S I M I L, Sistema de Información Micológica Ibérica en Línea. Real Jardín Botánico de Madrid, C.S.I.C. Proyecto Flora Micológica Ibérica I-VI (19902008). Ministerio de Educación y Ciencia, España. http://www.rjb.csic.es/fmi/sim.php (last up. 06/2008).

Kirk PM, Cannon PF, David JC, Stalpers JA. 2001. Ainsworth \& Bisby's Dictionary of the Fungi. ${ }^{\text {th }}$ edition. CABI Publishing, Oxford University Press, U.K.

Kirk PM. 2004-08. Index Fungorum. http://www.speciesfungorum.org/Index.htm (last up. 06/2008).

Mendes JC, Queiroz DX, Anastácio PA, Gonçalves MT, Cardoso MR, Coelho MG. 1991. Normais Climatológicas da região de "Alentejo e Algarve”, correspondentes a 1951-1980. O Clima de Portugal, 49(4): 35-36.

Pinho-Almeida F, Baptista-Ferreira JL. 1996. Macromicetos da Herdade da Ribeira Abaixo (Grândola). Portugalia Acta Biologica, Sér. B, Sist. 17: 155-183.

Pinho-Almeida F, Baptista-Ferreira JL. 2005. Cogumelos da Barrosinha (Alcácer do Sal) Inventariação de macrofungos em áreas seleccionadas. Centro de Micologia da Universidade de Lisboa, Lisboa.

SMM. 2008. Portal de la Sociedad Micológica de Madrid. España. http.www.socemicolmadrid.org (last up. 06/2008). 